\title{
EXISTENCE THEOREMS FOR NONSELFADJOINT SEMILINEAR ELLIPTIC BOUNDARY VALUE PROBLEMS
}

\author{
LAMBERTO CESARI \\ Department of Mathematics, University of Michigan, Ann Arbor, Michigan 48109, U.S.A \\ and \\ PatRIzIA PUCCI* \\ Dipartimento di Matematica, Università degli Studi, 06100 Perugia, Italy
}

(Received 1 April 1984; received for publication 25 February 1985)

Key words and phrases: Existence of solutions, nonlinear elliptic partial differential equations, nonselfadjoint boundary value problems, the alternative method, square norm topology, finite dimensional kernels and cokernels.

\section{INTRODUCTION}

IN THE present paper we present new existence theorems for weak and strong solutions of problems of the form

$$
E x=N x,
$$

where $E$ is a real elliptic linear differential operator in a bounded domain $G$ of $\mathbb{R}^{n}$ with a given system of linear homogeneous conditions, say, $B x=0$ on the boundary $\partial G$ of $G$ and where $N$ is a Nemitsky type nonnecessarily linear operator.

We shall make use here of the alternative method, and particularly we shall make use for the elliptic case of new remarks. These remarks suggest that both the auxiliary and bifurcation equations can be analyzed under different topologies, and by a more specific construction of the operator $S: Y_{0} \rightarrow X_{0}$.

Actually some of these remarks have been already used implicitly in previous papers on the semilinear wave equation in $\mathbb{R}^{2}$ (Cesari and Kannan [5], Cesari and Pucci [6]).

For selfadjoint elliptic problems, Landesman and Lazer [9] proved, also by the alternative method, a remarkable theorem which was then extended by Williams [14] by the same method, and by others by different arguments. Later, Shaw [12] proved, again by the alternative method, that Landesman's and Lazer's theorem extends even to nonselfadjoint problems with equal Fredholm indices and whose eigenfunctions share regions of positivity and negativity with their corresponding adjoint eigenfunctions.

In the present paper we definitely aim at elliptic problems which are not necessarily selfadjoint and do not necessarily satisfy Shaw's requirements. The sufficient conditions we obtain are more quantitative in character and concern the cases $N x=f(t)+g\left(t, D^{\alpha} x\right)$ and $N x=f(t)+g(t, x(t)), t \in G$. However, as we show by examples for the case $N x=f(t)+$ $g(t, x(t))$, our sufficient conditions for existence allow a great freedom on $g$, on which no monotonicity is required.

* This work was done within the Gruppo Nazionale per l'Analisi Funzionale e le sue Applicazioni del Consiglio Nazionale delle Ricerche, Rome, Italy. 


\section{GENERAL ASSUMPTIONS}

Let $G$ be a bounded connected open set in $\mathbb{R}^{n}$ with smooth boundary $\partial G$; in fact most of our results extend to a compact connected smooth Riemannian $n$-manifold with or without boundary. On the other hand, we shall consider situations where the smoothness assumptions on $\partial G$ can be relaxed so as to allow, say, $G$ to be an interval in $\mathbb{R}^{n}$.

For $s$ a nonnegative integer we denote by $H^{s}$ the Sobolev (Hilbert) space $W^{5.2}(G)$ of square integrable functions on $G$ whose (distributional) derivatives of order $\leqslant s$ are also square integrable functions $\left(H^{0}=L_{2}(G)\right)$, with norm $\|x\|_{H^{s}}=\left(\sum_{0 \leqslant, a \mid \leqslant s}\left\|D^{a} x\right\|_{2}^{2}\right)^{1 / 2}$. Here we denote by $(x, y)$ and $\|x\|_{2}=(x, x)^{1 / 2}$ the inner product and norm in $L_{2}(G)$. Then $H_{0}^{s}$ is the linear subspace of $H^{s}$ which is obtained by completion in the norm above of the set of the functions of class $C^{\infty}$ and compact support in $G$.

Let $E$ be a (not necessarily selfadjoint) uniformly elliptic real linear partial differential operator of order $2 m$, i.e.

$$
E x=\sum_{\alpha|,| \beta \mid \leq m}(-1)^{|\beta|} D^{\beta}\left(a_{\alpha \beta}(t) D^{\alpha} x\right)
$$

where $\alpha=\left(\alpha_{1}, \ldots, \alpha_{n}\right), \beta=\left(\beta_{1}, \ldots, \beta_{n}\right),|\alpha|=\alpha_{1}+\ldots+\alpha_{n}$, all $\alpha_{s}, \beta_{s}$ are nonnegative integers, with associated linear homogeneous boundary conditions, say $B x=0$ on $\partial G$; or more explicitly $B_{j}\left(t, D^{\alpha} x\right)=0, j=1, \ldots, M$, where $D^{\alpha} x$ denotes the set of the derivatives $D^{a} x$ on the boundary $\partial G, 0 \leqslant|\alpha| \leqslant K \leqslant m_{0}-1$ for some $K$ (the derivatives being replaced by their traces for $x$ in a Sobolev space $H^{m_{0}}$ ). We denote by $A[\phi, \psi]$ the usual bilinear form associated to $E$ :

$$
A[\phi, \psi]=\sum_{|\alpha|,|\beta| \leqslant m} \int_{G} a_{\alpha \beta}(t) D^{\alpha} \psi(t) D^{\beta} \phi(t) \mathrm{d} t .
$$

We denote by $E^{*}$ the formal adjoint operator,

$$
E^{*} y=\sum_{a|.| \beta \leqslant m}(-1)^{|\beta|} D^{\beta}\left(a_{\beta a}(t) D^{\alpha} y\right)
$$

with the associated boundary conditions, say $D y=0$ on $\partial G$, or $D_{s}\left(t, D^{\beta} y\right)=0, s=1, \ldots$, $N$.

We assume that both $\operatorname{Ker} E$ and $\operatorname{Ker} E^{*}$ are finite dimensional, with $x>p \geqslant q \geqslant 0, p=$ $\operatorname{dim} \operatorname{Ker} E, q=\operatorname{dim} \operatorname{Ker} E^{*}$. (However, we shall consider situations where $x=p>q \geqslant 0$ ).

A few words on the concept of solution $x$ of the linear problem $E x=f, f \in L_{2}(G)$, with $B_{i} x=0, j=1, \ldots, M$.

For weak solutions we need only to assume that the coefficients $a_{\alpha \beta}$ are measurable bounded functions on $G$. For instance, for the typical homogeneous Dirichlet boundary problem the boundary conditions are given by $x=0, \partial x / \partial n=0, \ldots, \partial^{m-1} x / \partial n^{m-1}=0, n$ the exterior normal to $\partial G$, and we say that $x$ is a weak solution of $E x=f$ provided $x \in I_{0}^{m}$ and $A[x, \psi]=$ $(f, \psi)$ for all $\psi \in C_{0}^{x}(G)$. In other words, $W=D(E)=H_{0}^{m}$ is the domain of $E$. We refer to [2] for the concept of weak solution for more general boundary value problems.

For strong, or classical solutions, we need to assume that the coefficients $a_{\alpha \beta}$ are of class $C^{i \beta \mid}(\tilde{G})$. Again, for the homogeneous Dirichlet boundary value problem above we say that $x$ 
is a strong solution of $E x=f$ provided $x \in H_{0}^{m} \cap H^{2 m}$ and $E x=f$ a.e. in $G$. Thus, $W=$ $D(E)=H_{0}^{m} \cap H^{2 m}$ and $W$ is equipped with the topology of $H^{2 m}, W=D(E)$.

In general, let $W=D(E)$ be the subspace of all elements $x$ of a Sobolev space $H^{m_{0}}$ for which $B x=0$, and we assume that $W$ is closed in $H^{m_{0}}$. Since $B$ involves derivatives of orders $\leqslant K$ we shall assume $K \leqslant m_{0}-1$. For weak solutions of the Dirichlet problem above we have $K=m-1, m_{0}=m$. For strong solutions of the same problem we have $K=m-1, m_{0}=$ $2 m$.

We assume that a Fredholm's alternative theorem holds, that is, for $f \in L_{2}(G)$, the problem $E x=f$, with $B_{j} x=0, j=1, \ldots, M$, has a solution $x \in W$ if and only if $f$ is orthogonal (in $\left.L_{2}(G)\right)$ to all elements $\omega$ of $\operatorname{Ker} E^{*}$.

Let $X=Y=L_{2}(G)$. We assume that $E$ is a closed operator with range $R(E)$ closed in $Y$.

All the assumptions above are generally true for reasonable boundary conditions $B$ (cf., e.g., $[10$, pp. $148-154,111-113])$.

Let $N$ be a (nonlinear) Nemitsky operator of the form $N x=f(t)+g\left(t, D^{a} x\right)$, where $\int$ is a given element in $L_{2}(G)$, where $D^{\alpha} x$ denotes the set of all derivatives of $x$ of orders $\alpha, 0 \leqslant|\alpha|$ $\leqslant k_{0}$, where $k_{0}$ is any integer $\leqslant 2 m$ and in any case $k_{0} \leqslant m_{0}$, and where $g(t, u)$, or $g: G \times \mathbb{R}^{\mu}$ $\rightarrow \mathbb{R}$, is measurable in $t$ for all $u \in \mathbb{R}^{\mu}$, and continuous in $u$ for a.a. $t \in G$. Here $\mu=1+n+$ $n(n+1) / 2+\ldots+n\left(n+k_{0}-1\right) / 2$ is the number of different derivatives in $\mathbb{R}^{n}$ of orders $\alpha$, $0 \leqslant|\alpha| \leqslant k_{0}$.

Thus, for $g$ bounded in $G \times \mathbb{R}^{\mu}$, then since $0 \leqslant k_{0} \leqslant m_{0}$, and $W \subset H^{m_{0}} \subset H^{k_{0}}$, then $N: W \rightarrow H^{0}$ is a bounded continuous operator from $W \subset H^{m_{0}}$ to $H^{0}=L_{2}(G)$ in the topologies of $H^{m_{0}}$ and $L_{2}(G)$ (cf. [13, p. 155; 8, p. 27]). Again, for $g$ bounded, the range $R(N)$ of $N$ is made up of functions on $G$ which are in absolute value $\leqslant|f(t)|+\sup |g|$. If both $f$ and $g$ are bounded, then the range of $N$ is a subset of $L_{x}(G)$, and $N$ is a bounded continuous operator from $W$ to $L_{x}(G)$.

If $g=g(t, u)$ is bounded in $G \times \mathbb{R}^{\mu}$ and uniformly Lipschitzian in $u$, then $N: W \rightarrow H^{0}$ is a Lipschitzian operator.

With these assumptions, we shall consider elliptic problems of the form:

$$
\left.\begin{array}{ll}
E x=f(t)+g\left(t, D^{\alpha} x\right), & t \in G, \\
B_{j}\left(t, D^{\alpha} x\right)=0 \quad \text { on } \partial G, & j=1, \ldots, M .
\end{array}\right\}
$$

\section{SOME FURTHER PROPERTIES OF $E$}

Let $\phi_{1}, \ldots, \phi_{p}$ be an orthonormal basis for Ker $E$, the elements $\phi_{i}$ being certainly in $X=L_{2}(G)$, and let $P: X \rightarrow X$ denote the orthogonal projection of $X$ onto $X_{0}=\operatorname{Ker} E=$ $\left\langle\phi_{1}, \ldots, \phi_{p}\right\rangle$ defined by $P x=\sum_{i=1}^{p}\left(x, \phi_{i}\right) \phi_{i}$ for $x \in X$. Then $P P=P$ and we take $X_{1}=$ $(\operatorname{Ker} E)^{\perp}=(I-P) X$ so that we have the decomposition $X=X_{0}+X_{1}, X_{0}=\operatorname{Ker} E$.

The restriction of $E: D(E) \subset X \rightarrow Y$ to the subspace $D(E) \cap(\operatorname{Ker} E)^{\perp}$ is a $1-1$ closed linear operator, whose closed range is the complete range of $E$. Hence, by the closed graph theorem, the inverse map $H=\left[E \mid D(E) \cap(\operatorname{Ker} E)^{\perp}\right]^{-1}$ is a $1-1$ continuous linear operator with domain $R(E)$ and range $D(E) \cap(\operatorname{Ker} E)^{\perp}$, or $D(H)=R(E), R(H)=D(E) \cap(\operatorname{Ker} E)^{\perp}$. 
Moreover,

$$
\left.\begin{array}{ll}
E H y=y & \text { for all } y \in R(E), \\
H E x=x-\sum_{i=1}^{p}\left(x, \phi_{i}\right) \phi_{i} & \text { for all } x \in D(E), \\
\|H y\|_{w} \leqslant L\|y\|_{2} & \text { for all } y \in R(E) \text { and some constant } L .
\end{array}\right\}
$$

Let $\omega_{1}, \ldots, \omega_{q}$ be an orthonormal basis for $\operatorname{Ker} E^{*}$, the elements $\omega_{s}$ being certainly in $Y=L_{2}(G)$, and let $Q: Y \rightarrow Y$ denote the orthogonal projection of $Y$ onto $Y_{0}=\operatorname{Ker} E^{*}=$ $\left\langle\omega_{1}, \ldots, \omega_{q}\right\rangle$ defined by $Q y=\sum_{s=1}^{q}\left(y, \omega_{s}\right) \omega_{s}$ for $y \in Y$. Then, $Q Q=Q$ and we take $Y_{1}=$ $\left(\operatorname{Ker} E^{*}\right)^{\perp}=(I-Q) Y$ so that we have the decomposition $Y=Y_{0}+Y_{1}$.

Thus, by the Fredholm alternative theorem, for every $f \in Y=L_{2}(G)$ there is a solution of $E x=f$ if and only if $f \perp \operatorname{Ker} E^{*}$, that is, if and only if $Q f=0$, that is, if and only if $f \in Y_{1}$, with $Y_{1}=(I-Q) Y=\left(\operatorname{Ker} E^{*}\right)^{\perp}$.

Since $R(E)=Y_{1}$ and $Q Y_{1}=0$, then $Q E=0$; since $P X=\operatorname{Ker} E$ we also have $E P=0$. These two remarks and relations (2) yield now

$$
H E=I-P, \quad Q E=0=E P, \quad E H(I-Q)=I-Q,
$$

and these are only particularizations of the usual relations for the alternative method [4], namely:

$$
H(I-Q) E=I-P, \quad Q E=E P, \quad E H(I-Q)=I-Q .
$$

For $p>q$ we further decompose $X_{0}=\left\langle\phi_{1}, \ldots, \phi_{p}\right\rangle$ into a space, say $X_{01}=\left\langle\phi_{1}, \ldots, \phi_{q}\right\rangle$ of dimension $q$, and a space $X_{02}=\left\langle\phi_{q+1}, \ldots, \phi_{p}\right\rangle$ of dimension $p-q$. For $p=q$ we take $X_{01}=X_{0}$ and $X_{02}=\{0\}$.

We make here the specific assumption:

$$
\left.\begin{array}{l}
\text { the decomposition } X_{0}=X_{01}+X_{02} \text { can be made in such a way that the } q \times q \text { matrix } \\
M=\left[\left(\omega_{s}, \phi_{i}\right), s, i=1, \ldots q\right] \text { is nonsingular. }
\end{array}\right\}
$$

For instance, if $\phi_{i}=\omega_{i}, i=1, \ldots, q$, as in the selfadjoint case, then $M=I$ is the identity matrix. We shall use the notational convention to denote briefly by $\sigma$ any given vector $\sigma=\operatorname{col}\left(\sigma_{1}, \ldots, \sigma_{q}\right)$

We now define the linear map $S: Y_{0} \rightarrow X_{01}$. For any $y \in Y_{0}$ we have $y=\sum_{s=1}^{q} d_{s}^{\sharp} \omega_{s}$, with $d_{s}^{\#}=\left(y, \omega_{s}\right)$, since the $\omega_{s}$ form a basis, and we take $S y=\sum_{i=1}^{q} d_{i} \phi_{i}$, with $d=M^{-1} d^{\#}$. Let us prove that $S^{-1}(0)=\{0\}$. Indeed, if $y \in S^{-1}(0)$, then $S y=0$, that is $d=0$, hence $d^{\#}=0$, since $M$ is nonsingular, and $y=0$.

We note that $X_{01} \subset X=Y$; hence $Q: Y \rightarrow Y$ is well defined on $X_{01}$. Let us prove that $S Q x=$ $x$ for every $x \in X_{01}$. In other words, $S Q$ reduces to the identity map on $X_{01}$. Indeed, if $x \in$ $X_{01}$, then $x=\sum_{i=1}^{q} c_{i} \phi_{i}, c_{i}=\left(x, \phi_{i}\right)$, and 


$$
Q x=\sum_{s=1}^{q}\left(x, \omega_{s}\right) \omega_{s}=\sum_{s=1}^{q}\left(\sum_{i=1}^{q} c_{i} \phi_{i}, \omega_{s}\right) \omega_{s}=\sum_{s=1}^{q}\left(\sum_{i=1}^{q}\left(\omega_{s}, \phi_{i}\right) c_{i}\right) \omega_{s}=\sum_{s=1}^{q} c_{s}^{*} \omega_{s},
$$

where $c^{\#}=M c$, and then $S Q x=\sum_{i=1}^{q} c_{i}^{\prime} \phi_{i}$, with $c^{\prime}=M^{-1}(M c)=c$, or $S Q x=x$.

It may occur that a given element, say $\omega_{1}$ in $Y_{0}$ is also an element, say $\phi_{1}$ in $X_{01}$. Since both bases are orthonormal, the matrix $M=\left[m_{i j}\right]$ has $m_{11}=1$, and all $m_{1 i}=m_{i 1}=0$ for $i \neq 1$. In particular, if $\phi_{i}=\omega_{i}, i=1, \ldots, q$, as in the selfadjoint case, then $M=I$ is the $q \times q$ identity matrix as already stated.

\section{THE INTERMEDIATE TOPOLOGY}

As we know from the alternative method (cf. [4]), the original problem $E x=N x$, with the conditions $B x=0$ on $\partial G$, is equivalent to the system of auxiliary and bifurcation equations:

$$
x=P x+H(I-Q) N x, \quad Q N x=0, \quad x \in W,
$$

This system can be written as the problem of the fixed points of the transformation $\mathscr{T}:\left(x_{01}\right.$, $\left.x_{02}, x_{1}\right) \rightarrow\left(\bar{x}_{01}, \bar{x}_{02}, \bar{x}_{1}\right)$ defined by

$$
\mathcal{J}: \begin{aligned}
& \bar{x}_{1}=\mathscr{J}_{1} x=H(I-Q) N\left(x_{01}+x_{02}+x_{1}\right), \\
& \bar{x}_{01}=\mathscr{T}_{0} x=x_{01}-k \operatorname{SQN}\left(x_{01}+x_{02}+x_{1}\right), \quad \bar{x}_{02}=x_{02} .
\end{aligned}
$$

where $x=x_{01}+x_{02}+x_{1} \in W, \bar{x}=\bar{x}_{01}+\bar{x}_{02}+\bar{x}_{1} \in W, x_{01}, \bar{x}_{01} \in X_{01} \subset W, x_{02}, \bar{x}_{02} \in X_{02} \subset$ $W, x_{1}, \bar{x}_{1} \in X_{1} \cap W$, and the chain of maps is as follows

$$
\begin{gathered}
\mathscr{T}_{1}: W \stackrel{N}{\rightarrow} L_{2}(G) \stackrel{I-Q}{\rightarrow} Y_{1} \stackrel{H}{\rightarrow} X_{1} \cap W, \\
\mathscr{T}_{0}: W \stackrel{N}{\rightarrow} L_{2}(G) \stackrel{Q}{\rightarrow} Y_{0} \stackrel{s}{\rightarrow} X_{01} \subset W,
\end{gathered}
$$

where $X_{01}$ is a finite dimensional subspace of $W$. Thus, $\mathscr{T}$ can be thought of as a map from $W$ into $W, W \subset H^{m_{0}}, W$ with the topology of $H^{m_{0}}$.

We shall introduce a different topology.

Indeed, we shall denote by $Z$ a Banach space satisfying the following requirements:

$$
\left.\begin{array}{l}
W \subset H^{m_{0}} \subset Z \subset H^{k_{0}} \subset Y=L_{2}(G) \text { with continuous imbeddings } j_{1}: W \rightarrow Z . \\
j_{2}: Z \rightarrow H^{k_{0}}, \text { and } j_{1} \text { compact. }
\end{array}\right\}
$$

Since $j_{3}: H^{k_{0}} \rightarrow L_{2}(G)$ is certainly continuous, then $j_{3} j_{2}: Z \rightarrow L_{2}(G)$ is also continuous. Note that for the continuity of $j_{1}$ and $j_{2}$ we require that for some constants $\gamma, \beta$ we have $\|x\|_{Z} \leqslant \gamma\|x\|_{W}$ for all $x \in W$, and $\|x\|_{H^{k_{0}}} \leqslant \beta\|x\|_{Z}$ for all $x \in Z$.

Under assumptions (5) the chain of maps in the transformation $\mathscr{T}$ becomes

$$
\left.\begin{array}{c}
\mathscr{T}_{1}: Z \stackrel{i_{2}}{\rightarrow} H^{k_{0}} \stackrel{N}{\rightarrow} L_{2}(G) \stackrel{I-Q}{\rightarrow} Y_{1} \stackrel{H}{\rightarrow} X_{1} \cap W \stackrel{j_{1}}{\rightarrow} X_{1} \cap Z \\
\stackrel{j_{2}}{\mathscr{J}_{2}}: Z \stackrel{N}{\rightarrow} H^{k_{0}} \stackrel{\sim}{\rightarrow} L_{2}(G) \stackrel{Q}{\rightarrow} Y_{0} \stackrel{s}{\rightarrow} X_{01} \stackrel{j_{1}}{\rightarrow} X_{01} \subset Z
\end{array}\right\}
$$

where $X_{01}$ is a finite dimensional subspace of $W$. 
As a particular case we assume first that $2\left(m_{0}-k_{0}\right)>n$ so that, from Sobolev's imbedding theorem (cf., e.g., [1, p. 97, Case C]), for any $x \in W \subset H^{m_{0}}$, the function $x$ and all its distributional derivatives $D^{\alpha} x, 0 \leqslant|\alpha| \leqslant k_{0}$, are bounded in $G$, that is, are in $L_{x}(G)$, and are continuous in the open set $G$. Then we can take for instance $Z=H^{k_{0}}$, or $Z=W^{k_{0} \cdot{ }^{x}}(G)$, since in either case the imbeddings

$$
\begin{aligned}
& j_{1}: W \subset H^{m_{0}} \rightarrow H^{k_{0}}, \quad j_{2}: H^{k_{0}} \rightarrow H^{k_{0}} \text { the identity, or } \\
& j_{1}: W \subset H^{m_{0}} \rightarrow W^{k_{0} \cdot x}(G), \quad j_{2}: W^{k_{0} \cdot x}(G) \rightarrow H^{k_{0}},
\end{aligned}
$$

are continuous, and $j_{1}$ is compact.

In the further particular case in which $k_{0}=0,2 m_{0}>n$, then $g=g(t, x)$ depends only on $x=x(t)$ and not on the derivatives, and for any $x \in W \subset H^{\left.m_{0}\right)}, x$ is a bounded function on $G$, or $x \in L_{x}(G)$, (hence, $\operatorname{Ker} E \subset L_{x}(G)$ ), and we can take $Z=L_{x}(G)$. Moreover, we assumed $g$ to be bounded, and for $f=f(t)$ in (1) also bounded, then $N: L_{x}(G) \rightarrow L_{x}(G)$. Furthermorc, we assume that $\operatorname{Ker} E^{*}$ is made up of bounded functions. Now we may restrict $X=Y=$ $L_{2}(G)$ to the space $X^{*}=Y^{*}=L_{x}(G)$ with the norm $\|x\|_{x_{*}}=\sup |x(t)|$ of $L_{x}(G)$. Let $X_{0}^{*}=$ $P X^{*}, X_{1}^{*}=(I-P) X^{*}, Y_{0}^{*}=Q Y^{*}, Y_{1}^{*}=(I-Q) Y^{*}$, and now $\operatorname{Ker} E$ and $\operatorname{Ker} E^{*}$ are made up of bounded functions (all $\phi_{i}$ and $\omega_{s}$ are bounded in $G$ ), and thus $X_{0}^{*}, Y_{0}^{*} \subset L_{x}(G)$. In this situation then relations (5) become

$$
\left.\begin{array}{l}
W \subset H^{m_{0}} \rightarrow Z=Y=L_{2}(G) \text { with continuous imbeddings } j_{1}: W \rightarrow Z=L_{x}(G), \\
j_{2}: L_{x}(G) \rightarrow L_{x}(G) \text { the identity, and } j_{1} \text { is compact. }
\end{array}\right\}
$$

Moreover, the chain of maps in $\mathscr{T}$ becomes:

$$
\left.\begin{array}{l}
\mathscr{T}_{1}: L_{x}(G) \stackrel{N}{\rightarrow} L_{x}(G) \stackrel{I-Q}{\rightarrow} Y_{1}^{*} \stackrel{H}{\rightarrow} X_{1}^{*} \cap W \stackrel{j_{1}}{\rightarrow} X_{1}^{*} \cap L_{x}(G) \\
\mathscr{T}_{0}: L_{x}(G) \stackrel{N}{\rightarrow} L_{x}(G) \stackrel{Q}{\rightarrow} Y_{0}^{*} \stackrel{S}{\rightarrow} X_{01}^{*} \cap W \rightarrow X_{01}^{*} \subset L_{x}(G) .
\end{array}\right\}
$$

and thus $\mathscr{T}$ is a map from $L_{x}(G)$ into $L_{x}(G)$.

On the other hand, the operators $P: X^{*} \rightarrow X^{*}, Q: Y^{*} \rightarrow Y^{*}, H: Y_{0}^{*} \rightarrow X_{1}^{*}$, $S Q: Y_{0}^{*} \rightarrow X_{0}^{*}$ should be thought of in the topology of $L_{x}(G)$, and the norms of $P$ and $Q$ may be $\geqslant 1$, and the norm of $H$ may be different from $L$. We may not need the exact value of these norms but estimates, say

$$
\|P\| \leqslant c_{1}, \quad\|I-P\| \leqslant c_{2}, \quad\|Q\| \leqslant c_{3}, \quad\|I-Q\| \leqslant c_{4}, \quad\|H(I-Q)\| \leqslant L_{0}, \quad\|S Q\| \leqslant c_{5} .
$$

and certainly $c_{1}, c_{2}, c_{3}, c_{4}, c_{5} \geqslant 1$.

\section{EXISTENCE THEOREMS}

The general assumptions in Section 2 are typical of elliptic problems and we do not repeat them here.

Let us write $g=g\left(t, D^{\alpha} x, 0 \leqslant|\alpha| \leqslant \mu\right)$ in the form $g\left(t, x, D^{a} x, 1 \leqslant|\alpha| \leqslant \mu\right)$, that is, $g=$ $g(t, u), u \in \mathbb{R}^{\prime \prime}$, in the form $g=g(t, z, \zeta), z \in \mathbb{R}, \zeta \in \mathbb{R}^{\mu-1}, t \in G$. We shall denote by $L_{0}, c_{5}$ positive constants so that

$$
\|H(I-Q) y\|_{Z} \leqslant L_{0}\|y\|_{2}, \quad\|S Q y\|_{Z} \leqslant c_{5}\|y\|_{2} \text { for all } y \in L_{2}(G) .
$$

Note that $L_{0} \leqslant \gamma L$ since $\|H(I-Q) y\|_{z} \leqslant \alpha\|H(I-Q) y\|_{2} \leqslant \alpha L\|y\|$. 
THEOREM 1. Under assumptions (3) and (5) with $W \subset Z \subset L_{2}(G), j_{1}: W \rightarrow Z, j_{2}: Z \rightarrow$ $H^{k_{0}}(G)$ continuous and $j_{1}$ compact, let $p=q, f \in L_{2}(G), g: G \times \mathbb{R}^{\mu} \rightarrow \mathbb{R}$ and assume that for suitable positive constants $c, C, D, R_{0}, r, \rho, k$ we have

$$
\begin{aligned}
& \|f\|_{2} \leqslant c, \quad|g(t, u)| \leqslant|G|^{-1 / 2} C \quad \text { for }(t, u) \in G \times \mathbb{R}^{\mu}, \\
& |g(t, u)-g(t, v)| \leqslant D|u-v| \quad \text { for a.a. } t \in G \text { and } u, v \in \mathbb{R}^{\mu}, \\
& \left\|x(t)-k g\left(t, x(t),\left(D^{\alpha} x\right)(t), 1 \leqslant|\alpha| \leqslant k_{0}\right)\right\|_{2} \leqslant \rho R_{0} \text { for } x \in Z \text { and }\|x\|_{Z} \leqslant R_{0}, \\
& L_{0}(c+C) \leqslant r, \rho c_{5}<1, \\
& k c_{5}(c+D \beta r) \leqslant\left(1-\rho c_{5}\right) R_{0} .
\end{aligned}
$$

Then, problem (1) has at least a solution $x \in W$ with $\|x\|_{Z} \leqslant R_{0}+r$.

If $p>q$, then for every element $\xi_{02} \in X_{02} \subset \operatorname{Ker} E \subset W$ we take $x=\xi_{02}+y$ so that problem $E x=f(t)+g\left(t, D^{\alpha} x\right), B x=0$, is changed into $E y=\tilde{f}(t)+\tilde{g}(t, D y), B y=0$, where $\tilde{f}=$ $f-E \xi_{02}=f$ since $\xi_{02} \in \operatorname{Ker} E$, and $g\left(t, D^{\alpha} y\right)=g\left(t, D^{\alpha} \xi_{02}+D^{\alpha} y\right)$. Now theorem 1 can be completed as follows.

If $p>q$ and there are constants $R_{2}>0$ and $c, C, D, R_{0}, r, \rho, k$ as above such that for every $\xi_{02} \in X_{02}$ with $\left\|\xi_{012}\right\|_{W} \leqslant R$, the new functions $\tilde{f}$ and $g$ satisfy relations (9)-(13), then problem (1) has $x-$ many solutions $x=x_{01}+\xi_{02}+x_{1}, x \in W \subset Z,\|x\|_{Z} \leqslant\left\|\xi_{02}\right\|_{Z}+R_{0}+r$, namely at least one for every $\xi_{02} \in X_{02}$ with $\left\|\xi_{02}\right\|_{W} \leqslant R_{2}$.

Proof. Let $\Omega=S_{0} \times S_{1}$ with $S_{0}=\left\{x_{01} \in X_{01}:\left\|x_{01}\right\|_{Z} \leqslant R_{0}\right\}, S_{1}=\left\{x_{1} \in X_{1}:\left\|x_{1}\right\|_{Z} \leqslant r\right\}$, where we note that $X_{01}$ is a finite dimensional subspace, $X_{01} \subset \operatorname{Ker} E \subset W \subset Z$, hence the topology in $X_{01}$ does not depend on the norm we choose. We have taken the norm of $Z$ in $S_{0}$ instead of the norm of $W$.

Now let us consider the transformation $\mathcal{T}$ in (4) with $x_{02}=0$. Hence, $\mathscr{T}$ is a transformation $\left(x_{01}, x_{1}\right) \rightarrow\left(\bar{x}_{01}, \bar{x}_{1}\right)$ which we think of as defined in $\Omega$. Now for $x=x_{01}+x_{1}, x_{01} \in S_{0}, x_{1} \in$ $S_{1}$, we have

$$
\begin{aligned}
& \bar{x}_{1}=\mathscr{T}_{1} x=H(I-Q)\left[f(t)+g\left(t,\left(D^{\alpha} x\right)(t)\right)\right] \\
& \left\|\bar{x}_{1}\right\|_{Z} \leqslant L_{0}\left(\|f\|_{2}+\left\|g\left(t, D^{a} x\right)\right\|_{2}\right) \leqslant L_{0}(c+C) \leqslant r .
\end{aligned}
$$

using hypotheses (8), (9) and (12).

Thus, $\mathscr{T}_{1}$ maps $\Omega$ into $S_{1}$. Moreover, by properties (6) and (8) we also have $\bar{x}_{1} \in W$.

We have now

$$
\begin{aligned}
\bar{x}_{01} & =\mathcal{T}_{0} x=x_{01}(t)-k S Q\left[f(t)+g\left(t,\left(D^{a} x\right)(t)\right)\right] \\
& =\left(x_{01}-S Q x_{01}\right)(t)+k S Q f(t)+S Q\left[x_{01}(t)-k g\left(t,\left(D^{\alpha} x_{01}\right)(t), 0 \leqslant|\alpha| \leqslant k_{0}\right)\right] \\
& +k S Q\left[g\left(t,\left(D^{a} x_{01}\right)(t), 0 \leqslant|\alpha| \leqslant k_{0}\right)-g\left(t,\left(D^{\alpha} x_{01}\right)(t)+\left(D^{\alpha} \bar{x}_{1}\right)(t), 0 \leqslant|\alpha| \leqslant k_{0}\right)\right],
\end{aligned}
$$

where the first term in the last expression is zero, since by using assumption (3) we have shown in Section 2 that $S Q$ is the identity map on $X_{01}$. Hence, we obtain

$$
\begin{aligned}
\left\|\bar{x}_{01}\right\|_{Z} & \leqslant k c_{5} c+c_{5} \rho R_{0}+k c_{5} D \beta\left\|\bar{x}_{1}\right\|_{Z} \\
& \leqslant k c_{5} c+c_{5} \rho R_{0}+k c_{5} D \beta r \leqslant R_{0},
\end{aligned}
$$

making use of assumptions (8), (9), (11), (10) and (13). 
Thus, $\mathscr{T}_{0}$ maps $\Omega$ into $S_{0}$, and $\mathscr{T}$ maps $\Omega$ into itself.

Let us prove that $\mathscr{T}: \Omega \rightarrow \Omega$ is a compact map. Indeed, $\mathscr{T}_{1} \Omega$ is a bounded closed subset of $W$, and this set is then compact in $Z$ because $j_{1}$ is a compact map by assumption (5). On the other hand, by relations (6) we note that $\mathscr{T}_{0} \Omega$ is a bounded closed subset of Ker $E$ which is a finite dimensional space. Thus, $G \Omega$ is a compact set in $Z$.

By Schauder's fixed point theorem, $\mathscr{T}: \Omega \rightarrow \Omega$ has a fixed point $x \in Z$ and actually $x \in W$ with $\|x\|_{Z} \leqslant R_{0}+r$ satisfying both the auxiliary and the bifurcation equation, and $x$ is a solution of the original problem (1).

In the particular case when $k_{0}=0,2 m_{0}>n$, then $g=g(t, x)$ depends only on the function $x=x(t)$ and not on its derivatives, and for $x \in W$, then $x=x(t)$ is bounded in $G$. As stated in Section 2, we can take $Z=L_{x}(G)$. In this situation, the following variant of theorem 1 is of interest.

We denote here by $L_{0}, c_{5}$ positive constants so that

$$
\|H(I-Q) y\|_{x} \leqslant L_{0}\|y\|_{x}, \quad\|S Q y\|_{x} \leqslant c_{5}\|y\|_{x} \quad \text { for all } y \in Y^{*}=L_{x}(G) .
$$

THEOREM 2. Under assumptions (3) and (5)' with $W \subset Z=L_{x}(G) \subset L_{2}(G), k_{0}=0,2 m_{0}>$ $n$, let $p=q, f \in L_{\times}(G), g: G \times \mathbb{R} \rightarrow \mathbb{R}$, and assume that for suitable constants $c, C, D, R_{0}$, $r, \rho, k$ we have

$$
\begin{aligned}
& \|f\|_{x} \leqslant c, \quad|g(t, u)| \leqslant C \quad \text { for }(t, u) \in G \times \mathbb{R} ; \\
& |g(t, u)-g(t, v)| \leqslant D|u-v|, \quad \text { for a.a. } t \in G, u, v \in \mathbb{R} \text { with }|u| \cdot|v| \leqslant R_{0}+r ; \\
& |u-k g(t, u)| \leqslant \rho R_{0}, \quad \text { for } t \in G \text { and }|u| \leqslant R_{0} ; \\
& L_{0}(c+C) \leqslant r, \quad \rho c_{5}<1 ; \\
& k c_{5}(c+D r) \leqslant\left(1-\rho c_{5}\right) R_{0} .
\end{aligned}
$$

Then, problem $E x=f(t)+g(t, x(t)), B x=0$, has at least a solution $x \in W$ with $\|x\|_{x} \leqslant R_{0}+$ $r$.

If $p>q$, then for every element $\xi_{02} \in X_{02} \subset \operatorname{Ker} E \subset W$, we take $x=\xi_{02}+y$ so that problem $E x=f(t)+g(t, x(t)), B x=0$, is changed into $E y=f(t)+\tilde{g}(t, y(t)) . B y=0$, where $\tilde{f}=f-E \xi_{02}=f$ since $\xi_{02} \in \operatorname{Ker} E, B y=0$, since certainly $B \xi_{02}=0$, and $\tilde{g}(t, y(t))=$ $g\left(t, \xi_{02}(t)+y(t)\right)$. Now theorem 2 can be completed as follows:

If $p>q$ and there are constants $R_{2}>0$ and $c, C, D, R_{0}, r, \rho, k$ as above such that, for every $\xi_{02} \in X_{02}$ with $\left\|\xi_{02}\right\|_{x} \leqslant R_{2}$ the new functions $\dot{f}$ and $\dot{g}$ satisfy relations (15)-(19), then problem $E x=f(t)+g(t, x(t)), B x=0$, has $x-$ many solutions $x=x_{01}(t)+\xi_{02}(t)+x_{1}(t)$, $x \in W \subset L_{x}(G),\|x\|_{x} \leqslant\left\|\xi_{02}\right\|_{x}+R_{0}+r$, namely at least one for every $\xi_{02} \in X_{02}$ with $\left\|\xi_{02}\right\|_{x} \leqslant$ $R_{2}$.

Proof. Let $\Omega=S_{0} \times S_{1}$ with $S_{0}=\left\{x_{01} \in X_{01}^{*}:\left\|x_{01}\right\|_{\infty} \leqslant R_{0}\right\}, S_{1}=\left\{x_{1} \in X_{1}^{*}:\left\|x_{1}\right\|_{\infty} \leqslant r\right\}$, with the same remarks as for theorem 1 . Now let us consider the transformation $\mathscr{T}$ in (4) (cf. (7)) with $x_{02}=0$. Hence, $\mathscr{T}$ is a transformation $\left(x_{01}, x_{1}\right) \rightarrow\left(\bar{x}_{01}, \bar{x}_{1}\right)$ which is defined in $\Omega$. As for 
theorem 1 we have now

$$
\begin{aligned}
& \bar{x}_{1}=\mathcal{J}_{1} x=H(I-Q)[f(t)+g(t, x(t))], \\
& \left\|\bar{x}_{1}\right\|_{x} \leqslant L_{0}\left(\|f\|_{x}+\|g(t, x(t))\|_{x}\right) \leqslant L_{0}(c+C) \leqslant r,
\end{aligned}
$$

by using assumptions (14), (15) and (18). Thus, $\mathscr{T}_{1}: \Omega \rightarrow S_{1}$ and analogously, by (7) and (14), we obtain $\bar{x}_{1} \in W$ and $\left\|\bar{x}_{1}\right\|_{W} \leqslant L(c+C)$.

On the other hand,

$$
\begin{aligned}
\bar{x}_{01} & =\mathscr{T}_{0} x=x_{01}(t)-k S Q[f(t)+g(t, x(t))] \\
& =\left(x_{01}-S Q x_{01}\right)(t)+k S Q f(t)+S Q\left[x_{01}(t)-k g\left(t, x_{01}(t)\right)\right] \\
& +k S Q\left[g\left(t, x_{01}(t)\right)-g\left(t, x_{01}(t)+\bar{x}_{1}(t)\right)\right]
\end{aligned}
$$

and

$$
\begin{aligned}
\left\|\bar{x}_{01}\right\|_{x} & \leqslant 0+k c_{5} c+c_{5} \rho R_{0}+k c_{5} D\left\|\bar{x}_{1}\right\|_{x} \\
& \leqslant k c_{5} c+c_{5} \rho R_{0}+k c_{5} D r \leqslant R_{0},
\end{aligned}
$$

by making use of assumptions (3), (14), (15), (17), (16) and (19).

Thus, $\mathscr{T}_{0}$ maps $\Omega$ into $S_{0}$, and $\mathscr{T}$ maps $\Omega$ into itself. Here $j_{1}: W \rightarrow L_{x}(G)$ is a compact map and therefore the compactness argument is the same as for the previous theorem 1 .

The case $p>q$ can be treated as before.

For problems of perturbation, that is, $E x=\varepsilon[f(t)+g(t, x(t))], B x=0$, where $\varepsilon>0$ is a small parameter, the following corollary holds:

CoROllary. Under assumptions (3) and (5) with $W \subset Z=L_{x}(G) \subset L_{2}(G), k_{0}=0,2 m_{0}>$ $n$, let $p=q, f \in L_{x}(G), g: G \times \mathbb{R} \rightarrow \mathbb{R}$, and assume that for suitable constants $C, D, R_{0}, r$, $\rho, k$ we have $|g(t, u)| \leqslant C$ for all $(t, u) \in G \times \mathbb{R} ;|g(t, u)-g(t, v)| \leqslant D|u-v|$ for all $t \in G$, $u, v \in \mathbb{R}$ with $|u|,|v| \leqslant R_{0}+r ;|u-k g(t, u)| \leqslant \rho R_{0}$ for all $t \in G$ and $|u| \leqslant R_{0} ; \rho c_{5}<1$. Then, there always are positive constants $c, \varepsilon_{0}, r_{0}$ such that for $\|f\|_{x} \leqslant c$, then $\varepsilon f$ and $\varepsilon g$ satisfy all relations (15)-(19) for $\varepsilon \leqslant \varepsilon_{0}$, and hence problem

$$
E x=\varepsilon[f(t)+g(t, x(t))], \quad B x=0,
$$

has at least a solution $x \in W$. with $\|x\|_{x} \leqslant R_{0}+r_{0} \varepsilon$, for every $\varepsilon \leqslant \varepsilon_{0}$.

For $p>q$ a statement analogous to the previous one holds.

Proof. First, $\varepsilon f, \varepsilon g$ satisfy relations (15), (16) with $c, C, D$ replaced by $\varepsilon c, \varepsilon C, \varepsilon D$ respectively and relation (17) with $k$ replaced by $\varepsilon^{-1} k$. Now we take $r_{0}=L_{0}(c+C)$ and we apply theorem 2 with $r$ replaced by $r^{\prime}=r_{0} \varepsilon=L_{0}(\varepsilon c+\varepsilon C)$, provided $r_{0} \varepsilon=L_{0}(\varepsilon c+\varepsilon C) \leqslant r$, that is, for $0<\varepsilon \leqslant \varepsilon_{1}=L_{0}^{-1}(c+C)^{-1} r$. Now relation (19) becomes

or

$$
\varepsilon^{-1} k c_{5}\left[\varepsilon c+\varepsilon D L_{0}(\varepsilon c+\varepsilon C)\right] \leqslant\left(1-\rho c_{5}\right) R_{0},
$$

$$
k c_{5}\left(1+\varepsilon D L_{0}\right) c+\varepsilon k D L_{0} C c_{5} \leqslant\left(1-\rho c_{5}\right) R_{0}
$$

and this relation can be satisfied by taking, say $\varepsilon \leqslant \varepsilon_{2}$ and

$$
\varepsilon_{2} \leqslant \min \left\{\left(2 k D L_{0} C c_{5}\right)^{-1}\left(1-\rho c_{5}\right) R_{0},\left(D L_{0}\right)^{-1}\right\}, c \leqslant\left(4 k c_{5}\right)^{-1}\left(1-\rho c_{5}\right) R_{0} .
$$

We shall take now $\varepsilon_{0}=\min \left[\varepsilon_{1}, \varepsilon_{2}\right]$. 


\section{EXAMPLES}

It is clear that theorems 1 and 2 hold for both selfadjoint and nonselfadjoint problems, though for selfadjoint problems the stronger theorem of Landesman and Lazer [9] holds. For the sake of simplicity, let us consider first two selfadjoint problems.

Example 1. Let us consider the elliptic problem

$$
\left.\begin{array}{l}
u_{t t}+u_{s s}+\left(2 \pi^{2} / T^{2}\right) u=f(t, s)+g(u(t, s)), \quad(t, s) \in G=[0, T] \times[0, T], \\
u(t, s)=0 \quad \text { on } \partial G .
\end{array}\right\}
$$

We know that the problem is strongly elliptic and selfadjoint. The operator $E$ defined by $E u=$ $u_{t t}+u_{s s}+\left(2 \pi^{2} / T^{2}\right) u$, with the homogeneous Dirichlet boundary conditions above, has eigenvalues and eigenfunctions

$$
\begin{aligned}
& \lambda_{a b}=\left(\pi^{2} / T^{2}\right)\left(2-a^{2}-b^{2}\right), \quad \phi_{a b}(t, s)=(2 / T) \sin (a \pi t / T) \sin (b \pi s / T), \\
& a, b=1,2, \ldots
\end{aligned}
$$

Also, $\operatorname{Ker} E=\left\{c \phi_{11}\right\}=\operatorname{Ker} E^{*}$, and thus we can take $\phi_{1}=\omega_{1}=(2 / T) \sin (\pi t / T)$ $\sin (\pi s / T), p=q=1$. Thus, $Q f=\left(f, \omega_{1}\right) \omega_{1}$, and for $f=\sum_{(a, b)} c_{a b} \phi_{a b}$, we have

$$
\|f\|_{2}=\sum_{(a, b)} c_{a b}^{2}, \quad(I-Q) f=\sum_{(a, b) \neq(1,1)} c_{a b} \phi_{a b}, \quad H(I-Q) f=\sum_{(a, b) \neq(1,1)} \lambda_{a b}^{-1} c_{a b} \phi_{a b} .
$$

To simplify notations, we take $\lambda_{a b}^{*}=2-a^{2}-b^{2}$, and we note that for $(a, b) \neq(1,1)$ these numbers have values $-3,-6,-8,-11, \ldots$ Hence

On the other hand

$$
\begin{aligned}
\|H(I-Q) f\|_{2} & =\left(\sum_{(a, b) \neq(1,1)} \lambda_{a b}^{-2} c_{a b}^{2}\right)^{1 / 2}=\left(T^{2} / \pi^{2}\right)\left(\sum_{(a, b) \neq(1,1)} \lambda_{a b}^{*-2} c_{a b}^{2}\right)^{1 / 2} \\
& \leqslant\left(T^{2} / \pi^{2}\right)(1 / 3)\left(\sum_{(a, b) \neq(1,1)} c_{a b}^{2}\right)^{1 / 2} \leqslant\left(T^{2} / 3 \pi^{2}\right)\|f\|_{2} .
\end{aligned}
$$

Thus,

$$
\begin{aligned}
|H(I-Q) f(t, s)| & \leqslant\left(T^{2} / \pi^{2}\right)\left(\sum_{(a, b) \neq(1,1)} \lambda_{a b}^{*-2}\right)^{1 / 2}\left(\sum_{(a, b) \neq(1,1)} c_{a b}^{2} \phi_{a b}^{2}\right)^{1 / 2} \\
& \leqslant\left(T^{2} / \pi^{2}\right)(2 / T)\left(\sum_{(a, b) \neq(1,1)} \lambda_{a b}^{*-2}\right)^{1 / 2}\|f\|_{2} \\
& \leqslant\left(T^{2} / \pi^{2}\right)(2 / T)\left(\sum_{(a, b) \neq(1,1)} \lambda_{a b}^{*-2}\right)^{1 / 2} T\|f\|_{\infty} .
\end{aligned}
$$

$$
\|H(I-Q) f\|_{x} \leqslant\left(2 T^{2} / \pi^{2}\right)\left(\sum_{(a, b) \neq(1.1)} \lambda_{a b}^{*-2}\right)^{1 / 2}\|f\|_{x} .
$$

For these $L_{2^{-}}$and $L_{\infty}$-estimates (cf. Cesari [3]).

The sum of the series can be evaluated by separating the terms with $1<a^{2}+b^{2}<50$, from those with $a^{2}+b^{2} \geqslant 50$, or

$$
\sum_{(a, b) \neq(1,1)} \lambda_{a b}^{*-2}=\left(\sum_{1<a^{2}+b^{2}<50}+\sum_{a^{2}+b^{2} \geqslant 50}\right) \lambda_{a b}^{*-2}=S_{1}+S_{2}
$$


For $a^{2}+b^{2} \geqslant 50$ we have $\left(a^{2}+b^{2}\right) /\left(a^{2}+b^{2}-2\right) \leqslant 50 / 48$ (the equality holds for $a=b=$ 5), and

$$
\begin{aligned}
S_{2} & =\sum_{a^{2}+b^{2} \geqslant 50}\left(a^{2}+b^{2}-2\right)^{-2} \leqslant(50 / 48)^{2} \sum_{a^{2}+b^{2} \geqslant 50}\left(a^{2}+b^{2}\right)^{-2} \\
& \leqslant(50 / 48)^{2} \sum_{a^{2}+b^{2} \geqslant 50} \int_{a-1}^{a} \int_{b-1}^{b}\left(t^{2}+s^{2}\right)^{-2} \mathrm{~d} t \mathrm{~d} s .
\end{aligned}
$$

It is immediately seen that for $a^{2}+b^{2} \geqslant 50$ we have $(a-1)^{2}+(b-1)^{2} \geqslant 32$ (again the equality holds for $a=b=5$ ), and

$$
\begin{aligned}
S_{2} & \leqslant(50 / 48)^{2} \iint_{t^{2}+s^{2} \geqslant 32}\left(t^{2}+s^{2}\right)^{-2} \mathrm{~d} t \mathrm{~d} s=(50 / 48)^{2} \int_{V_{32}}^{+\infty} \int_{0}^{\pi / 2} \rho^{-4} \rho \mathrm{d} \rho \mathrm{d} \theta \\
& =(50 / 48)^{2} 2^{-1}(32)^{-1}(\pi / 2)=(1.085068)(0.0245436)=0.026632 .
\end{aligned}
$$

There are only 29 terms in $S_{1}$ whose sum is 0.335210 . Hence

$$
S_{1}+S_{2}<0.335210+0.026632=0.361842 .
$$

Thus $(0.361842)^{1 / 2}=0.601533$, and

$$
\|H(I-Q) f\|_{x} \leqslant\left(T^{2} / \pi^{2}\right)(2)(0.361842)^{1 / 2}\|f\|_{x}=\left(T^{2} / \pi^{2}\right)(1.203066)\|f\|_{x} .
$$

We also have

$$
\begin{aligned}
|Q f(t, s)| & =\left|\left(\int_{0}^{T} \int_{0}^{T} f(\alpha, \beta) \phi_{11}(\alpha, \beta) \mathrm{d} \alpha \mathrm{d} \beta\right) \phi_{11}(t, s)\right| \\
& \leqslant(2 / T)\left(\int_{0}^{T} \sin (\pi \alpha / T) \mathrm{d} \alpha\right)\left(\int_{0}^{T} \sin (\pi \beta / T) \mathrm{d} \beta\right)\|f\|_{x}(2 / T) \\
& =(2 / T)(2 / T)(2 T / \pi)(2 T / \pi)\|f\|_{x}=\left(16 / \pi^{2}\right)\|f\|_{x} \\
& =(1.621139)\|f\|_{x} \\
|(I-Q) f(t, s)| & \leqslant(2.621139)\|f\|_{x},
\end{aligned}
$$

and if we take $S: Y_{0} \rightarrow X_{0}$ the identity map, we have

Thus, we can take

$$
|S Q f(t, s)| \leqslant(1.621139)\|f\|_{\infty} .
$$

$$
L_{0}=\left(T^{2} / \pi^{2}\right)(1.203066), \quad c_{4}=2.621139, \quad c_{5}=1.621139 .
$$

For instance, for $c=0.1, C=1, D=2, T=0.5, k=1, R_{0}=1, \rho=0.4$, relations (15)-(19) are satisfied since

$$
\begin{gathered}
L_{0}(c+C)=\left(T^{2} / \pi^{2}\right)(1.203066)(0.1+1)=0.0335214<0.0336=r \\
\rho c_{5}=(0.4)(1.621139)=0.648455<1 \\
\left(1-\rho c_{5}\right) R_{0}=0.351544 \\
c_{5}(c+D r)=(1.621139)(0.1+0.0672)=0.271054<0.351544 .
\end{gathered}
$$

Thus, for any $f=f(t, s)$ measurable and bounded, $\|f\|_{x} \leqslant 0.1$, and any continuous $g=g(u)$ with $|g(u)| \leqslant 1,|g(u)-g(v)| \leqslant 2|u-v|$ for all $|u|,|v| \leqslant 1.0336,|u-g(u)| \leqslant 0.4$ for $|u| \leqslant 1$, 


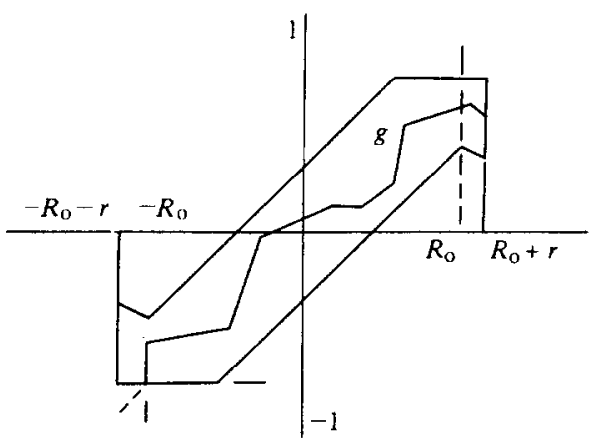

Fig. 1.

problem (20) has at least one solution. In other words, $g$ can be any continuous function, Lipschitzian of constant two, whose graph is within the heavy lines of Fig. 1.

Example 2. Let us consider the strongly elliptic and selfadjoint problem

$$
\left.\begin{array}{l}
u_{t t}+u_{s s}+5\left(2 \pi^{2} / T^{2}\right) u=f(t, s)+g(u(t, s)), \quad(t, s) \in G=[0, T] \times[0, T], \\
u(t, s)=0 \quad \text { on } \partial G .
\end{array}\right\}
$$

The operator $E$ defined by $E u=u_{t t}+u_{s s}+5\left(2 \pi^{2} / T^{2}\right) u$ with the Dirichlet homogeneous boundary conditions above has eigenvalues and eigenfunctions:

$$
\begin{aligned}
& \lambda_{\mathrm{ab}}=\left(\pi^{2} / T^{2}\right)\left(5-a^{2}-b^{2}\right), \quad \phi_{a \dot{b}}(t, s)=(2 / T) \sin (a \pi t / T) \sin (b \pi s / T), \\
& a, b=1,2, \ldots
\end{aligned}
$$

Also, Ker $E=\left\{c_{1} \phi_{12}+c_{2} \phi_{21}\right\}=\operatorname{Ker} E^{*}$ and thus we can take $\phi_{1}=\omega_{1}=\phi_{12}, \phi_{2}=\omega_{2}=$ $\phi_{21}, p=q=2$. Thus, $Q f=\left(f, \omega_{1}\right) \omega_{1}+\left(f, \omega_{2}\right) \omega_{2}$, and for $f=\sum_{(a, b)} c_{a b} \phi_{a b}$, we have

$$
\|f\|_{2}=\sum_{(a . h)} c_{a b}^{2}, \quad(I-Q) f=\sum^{\prime} c_{a b} \phi_{a b} .
$$

where $\Sigma^{\prime}$ ranges over all $(a, b)$ different from $(1,2)$ and $(2,1)$, and then

$$
H(I-Q) f=\sum^{\prime} \lambda_{a b}^{-1} c_{a b} \phi_{a b}
$$

and the smallest $\left|\lambda_{a b}\right|$ with $(a, b) \neq(1,2)$, and $(a, b) \neq(2.1)$ are $\lambda_{11}=\left(3 \pi^{2} / T^{2}\right), \lambda_{22}=$ $\left(-3 \pi^{2} / T^{2}\right)$. Hence

$$
\|H(I-Q) f\|_{2}=\left(\sum^{\prime} \lambda_{a b}^{-2} c_{a b}^{2}\right)^{1 / 2}\|f\|_{2} \leqslant\left(T^{2} / 3 \pi^{2}\right)\|f\|_{2}
$$

On the other hand, as before

$$
\|H(I-Q) f\|_{x} \leqslant\left(2 T^{2} / \pi^{2}\right)\left(\sum^{\prime} \lambda_{a b}^{*-2}\right)^{1 / 2}\|f\|_{x},
$$

where here $\lambda_{a b}^{*}=5-a^{2}-b^{2}$. 
The estimate of the sum of the series can be made as for example 1 . For $a^{2}+b^{2} \geqslant 50$ we have $\left(a^{2}+b^{2}\right) /\left(a^{2}+b^{2}-5\right) \leqslant 50 / 45=10 / 9$, and

$\sum_{a^{2}+b^{2}=50}\left(a^{2}+b^{2}-5\right)^{-2} \leqslant(10 / 9)^{2} \sum_{a^{2}+b^{2} \geq 50}\left(a^{2}+b^{2}\right)^{-2} \leqslant(1.234567)(0.0007669)=0.000947$

There are other 28 terms with $a^{2}+b^{2}<50$ and their sum is 0.385298 . Then,

$$
\sum^{\prime} \lambda_{a b}^{*-2} \leqslant 0.385298+0.000947=0.386245 \text {. }
$$

Thus, $(0.386245)^{1 / 2}=0.621486$, and

$$
\|H(I-Q) f\|_{x}<\left(T^{2} / \pi^{2}\right)(2)(0.386245)^{1 / 2}\|f\|_{x}=\left(T^{2} / \pi^{2}\right)(1.242972)\|f\|_{x} .
$$

Therefore, we can take

$$
L_{0}=\left(T^{2} / \pi^{2}\right)(1.242972), \quad c_{4}=2.621139, \quad c_{5}=1.621139 .
$$

For instance, for $c=0.1, C=1, D=2, T=0.5, k=1, R_{0}=1, \rho=0.4$, relations (15)-(19) are satisfied since

$$
\begin{gathered}
L_{0}(c+C)=\left(T^{2} / \pi^{2}\right)(1.242972)(1.1)=0.0346331<0.035=r \\
\rho c_{5}=(0.4)(1.621139)=0.648555<1 \\
\left(1-\rho c_{5}\right) R_{0}=0.351544 \\
c_{5}(c+D r)=(1.621139)(0.17)=0.275593<0.351544 .
\end{gathered}
$$

Thus, for any $f=f(t, s)$ measurable and bounded, $\|f\|_{x} \leqslant 0.1$, and any continuous $g=g(u)$ with $\left|g(u)_{x}\right| \leqslant 1,|g(u)-g(v)| \leqslant 2|u-v|$ for $|u|,|v| \leqslant 1.035,|u-g(u)| \leqslant 0.4$ for $|z| \leqslant 1$, problem (21) has at least one solution. A geometric picture of a possible $g$ is shown in Fig. 1.

Example 3. Let us consider the elliptic nonselfadjoint problem

$$
\begin{array}{cc}
E u \equiv u_{t t}+u_{s s}+2 \lambda^{2} u=f(t, s)+g(u(t, s)), & (t, s) \in G=[0, T] \times[0, T / 2], \\
u(0, s)=-\sigma\left(u_{t}(0, s)-u_{t}(T, s)\right), & 0 \leqslant s \leqslant T / 2, \\
u(T, s)=\sigma\left(u_{t}(0, s)-u_{t}(T, s)\right), & 0 \leqslant s \leqslant T / 2, \\
u(t, 0)=u(t, T / 2)=0, & 0 \leqslant t \leqslant T,
\end{array}
$$

where $\lambda=2 \pi / T$, and $\sigma>0$ is a given constant. For $\sigma=0$ we have the Dirichlet conditions $u=0$ on $\partial G$. The underlying linear problem is

$$
E u \equiv u_{t t}+u_{s s}+2 \lambda^{2} u=0
$$

with the same boundary conditions (23). The corresponding dual problem is then

$$
\begin{aligned}
E^{*} v & =v_{t}+v_{s s}+2 \lambda^{2} v=0, \quad(t, s) \in G, & & \\
v(0, s) & =v(T, s)=-\sigma\left(v_{t}(0, s)+v_{t}(T, s)\right), & & 0 \leqslant s \leqslant T / 2, \\
v(t, 0) & =v(t, T / 2)=0, & & 0 \leqslant t \leqslant T .
\end{aligned}
$$

The nonzero solutions of problem (24), (23) are all proportional to

$$
\Phi(t, s)=2^{3 / 2} T^{-1} \sin \lambda t \sin \lambda s, \quad(t, s) \in G
$$


the nonzero solutions of $(25),(26)$ are all proportional to

$$
\omega(t, s)=2^{3 / 2} T^{-1}\left(1+4 \sigma^{2} \lambda^{2}\right)^{-1 / 2}(\sin \lambda t-2 \sigma \lambda \cos \lambda t) \sin \lambda s
$$

In other words, $\operatorname{Ker} E=\{c \phi\}, \operatorname{Ker} E^{*}=\{\mathrm{d} \omega\}, p=q=1$. Certainly problem (22), (23) is nonselfadjoint and $\phi$ and $\omega$ do not share regions of positivity and negativity in $G$.

However, an analysis similar to the one for the preceding examples has been possible, leading to an existence theorem for weak solutions of problem (22), (23) under analogous requirements on $f$ and $g$. Also numerical examples have been exhibited. The intricate details, and the choice of topologies, will be presented elsewhere [11].

Example 4. Let us consider the elliptic nonselfadjoint problem of order $2 m=4$,

$$
\left.\begin{array}{l}
\Delta^{2} u=\varepsilon[f(t, s)+g(u(t, s))], \quad(t, s) \in G \\
B: \partial u / \partial x=0, \quad \partial(\Delta u) / \partial n=0 \quad \text { on } \partial G,
\end{array}\right\}
$$

where $\Delta=\partial^{2} u / \partial t^{2}+\partial^{2} u / \partial s^{2}, G$ is a bounded region in the $t s$-plane with smooth boundary $\partial G, n$ is the exterior normal to $\partial G$, and $\varepsilon$ is a small parameter, $\varepsilon>0$.

The linear operator $E=\Delta^{2}$ with the homogeneous boundary conditions above has $\operatorname{Ker} E=$ $\left\{c_{1}+c_{2} s+c_{3} s^{2}\right\}$, while Ker $E^{*}=\{c\}, c, c_{1}, c_{2}, c_{3}$ constants, $p=3, q=1$ (cf. Hormander [7, pp. 265-266]). Here we have $2 m=4$, and for weak solutions $u \in H_{0}^{2}$ we have $m_{0}=2, n=2$, $4>n$, so that for $f \in L_{2}(G)$ the solutions of the linear problem $\Delta^{2} u=f, B u=0$, are in $H_{0}^{2} \cap L_{x}(G)$. The corollary applies.

Upon evaluation of the constants $L_{0}$ and $c_{5}$, if a real function $g: \mathbb{R} \rightarrow \mathbb{R}$ satisfies $|g(u)| \leqslant C$ for all $|u| \leqslant R_{0}+r,|g(u)-g(v)| \leqslant D|u-v|$ for all $|u|,|v| \leqslant R_{0}+r,|u-k g(u)| \leqslant \rho R_{0}$ for $|u| \leqslant R_{0}, \rho c_{5}<1$, for given constants $C, R_{0}, r, D, \rho, k$, then there are constants $\varepsilon_{0}>0$, $c>0, r_{0}>0$, such that relations (15)-(19) of the corollary hold and problem (22) has at least one weak solution $u \in H_{0}^{2} \cap L_{x}(G)$ with $\|u\|_{\infty} \leqslant R_{0}+r \varepsilon_{0}$ for every $\varepsilon \leqslant \varepsilon_{0}$.

This example 4 is not selfadjoint, is not in the classes considered by Shaw in [12], and, for $g$ arbitrary and not differentiable, the usual theorems for perturbation type problems do not apply.

\section{REFERENCES}

1. Adams R. A., Sobolev Spaces, Academic Press, New York (1975).

2. Agmon S., Lectures on Elliptic Boundary Value Problems, Van Nostrand Math. Studies No. 2, Princeton (1965).

3. CESARI L., Function analysis and periodic solutions of nonlinear differential equations, Contrib. diff. Eqns 1 , $149-187$ (1963).

4. CESARI L., Functional Analysis, Nonlinear Differential Equations and the Alternative Method. Nonlinear Functional Analysis and Differential Equations (Edited by L. CeSARI, R. KANNAN and J. D. SCHuUR), pp. 1-196. Dekker, New York (1976).

5. Cesari L. \& Kannan R., Periodic solutions of nonlinear wave equations, Archs ration. Mech. Analysis 82 , 295312 (1983).

6. CESARI L. \& PUCCI P., Global periodic solutions of the nonlinear wave equation, Archs ration. Mech. Analysis (1984).

7. HoRmander L., Linear Partial Differential Operators, Springer, Berlin (1969).

8. Krasnosel'Ski M. A., Topological Methods in the Theory of Nonlinear Integral Equations, Macmillan, New York (1964).

9. Landesman E. M. \& LAzER A. C., Nonlinear perturbations of linear elliptic boundary value problems at resonance, J. Math. Mech. 19, 609-623 (1970). 
10. Lions J. L. \& Magenes E., Non-Homogeneous Boundary Value Problems and Applications. Vol. I. Springer. Berlin (1972).

11. PUCCI P., A semilinear elliptic problem which is nut selfadjoint, Archs ration. Mech. Analysis (to appear).

12. SHAw H. C., A nonlinear elliptic boundary value problem at resonance, J. diff. Eqns 26, 335-346 (1977).

13. Vainberg M., Variational Methods for the Study of Nonlinear Operators, Holden Day, San Francisco (1964).

14. Williams S., A sharp sufficient condition for solutions of a nonlinear elliptic boundary value problem, J. diff. Eqns 8, 580-586 (1970). 